\title{
GOBIERNO CORPORATIVO: ¿QUE ES? Y ¿COMO ANDAMOS POR CASA?
}

\author{
FERNANDO LeFORT
}

\begin{abstract}
This paper presents a simple framework in order to analyze different corporate governance mechanisms and their impact on firm structures and performance. Based on recent literature on the subject, the paper discusses the validity of Demsetz's evolution hypothesis on corporate governance structures and compares it to other hypothesis based on the recent empirical evidence at the international level. Specifically, the paper discusses the role of legal and regulatory systems in conditioning the development of different corporate governance structures. The paper emphasizes the issues related to corporate structure and governance in emerging economies. The paper also discusses the exogenous factors, such as regulations, that may help governance practices in the Chilean economy. In this context, the papers summarizes and discusses the growing literature on corporate governance and structure in Chile.
\end{abstract}

\section{RESUMEN}

Este artículo busca estructurar un marco conceptual sencillo que permita ordenar la discusión acerca de las diferentes formas de gobierno corporativo y de su efecto sobre la estructura y desempeño de las empresas. Basándose en la literatura reciente, el artículo discute la validez de la hipótesis de que en el largo plazo sobreviven las estructuras que tienden a mejorar el desempeño de las empresas y la compara con otras hipótesis alternativas tanto a nivel teórico como a la luz de la evidencia empírica. En particular, se discute el significativo

* Profesor Escuela de Administración, Pontificia Universidad Católica de Chile.

e-mail: flefort@faceapuc.cl

Keywords: Corporate Governance, Emerging Economies, Chile.

JEL Classification: G32 
rol de los sistemas legales y regulatorios en condicionar el desarrollo de las estructuras de gobierno corporativo en el mundo. En el marco de esta discusión, este artículo enfatiza aquellos aspectos que pueden ayudar a entender mejor el funcionamiento y efecto del gobierno corporativo en el caso de empresas que funcionan en economías emergentes. También se discute el tipo de factores exógenos, relacionados con regulaciones que pueden ayudar a mejorar las prácticas de gobierno corporativo en una economía emergente como la chilena. Con ese objetivo, se revisa y discute el grueso de la incipiente literatura académica sobre gobierno y estructura corporativa en Chile, a la luz del marco conceptual desarrollado previamente.

\section{INTRODUCCIÓN}

En los últimos 10 a 15 años la literatura académica en finanzas corporativas ha producido una larga lista de artículos analizando, tanto desde el punto de vista teórico como empírico, la relación que existe entre los diferentes aspectos que constituyen el gobierno de la corporación. Esta literatura ha analizado, además, la relación entre estos aspectos del gobierno corporativo y el desempeño de la empresa y de la economía. El ímpetu con el que esta literatura se ha desarrollado se debe a dos razones principalmente. Por un lado, responde al relativo consenso que los economistas han alcanzado respecto a la idea de que la teoría de la firma es en realidad muy compleja y no puede ser entendida a cabalidad mediante el uso de modelos tradicionales bajo condiciones de competencia e información perfecta. Por otro lado, este ímpetu se ha retroalimentado con la evidencia empírica que se ha acumulado apoyando algunas de las principales hipótesis en relación al gobierno de la corporación.

En términos simples, el gobierno corporativo consiste en el conjunto de relaciones que se establecen entre los diferentes participantes en la empresa con el fin de garantizar que cada uno reciba lo que es justo. Esto es crucial para proveer los incentivos adecuados para que se realicen las inversiones necesarias para el desarrollo de la empresa. La razón para que esto no ocurra en forma automática es la existencia de asimetrías de información y la imposibilidad de implementar contratos frente a cada una de las posibles eventualidades futuras.

Son muchos los aspectos que conforman el gobierno corporativo y condicionan, de una u otra forma, el desempeño de la empresa. Entre los aspectos propios a la empresa se destacan el sistema de toma de decisiones, la estructura de capital, los mecanismos de compensación a los ejecutivos y los sistemas de monitoreo. Entre los aspectos exógenos a la empresa son fundamentales el sistema legal, el mercado por el control corporativo, el mercado por los servicios gerenciales y el grado de competencia en los mercado de bienes e insumos que enfrenta la empresa. ${ }^{1}$

1 Ver Mathiesesen (2001) para una discusión acerca de los diferentes aspectos del gobierno corporativo. 
Estas diferentes dimensiones del gobierno corporativo pueden afectar el desempeño y valor de la empresa. Además, un adecuado gobierno corporativo favorece el desarrollo del sistema financiero y el crecimiento económico. ${ }^{2}$ Por otro lado, las diferentes dimensiones del gobierno corporativo se condicionan entre ellas. Por ejemplo, una estructura de toma de decisiones dominada por los miembros del directorio tenderá a favorecer un mayor endeudamiento que un sistema dominado por los ejecutivos. ${ }^{3}$ En algunos casos la causalidad entre estos aspectos del gobierno corporativo será bidireccional. Por ejemplo, un mayor porcentaje de la propiedad en manos de la administración de la empresa puede mejorar el desempeño de la empresa puesto que alinea los incentivos monetarios de los ejecutivos con los propietarios. ${ }^{4}$ Sin embargo, el desempeño económico de la empresa determinará a la larga la estructura de propiedad dominante, en el sentido de que las empresas con estructuras ineficientes tenderán a desaparecer con el paso del tiempo. ${ }^{5}$

Normalmente, el gobierno de la empresa implica establecer algún tipo de prioridad entre los diferentes stakeholders. En general, se asigna el control residual sobre los activos de la empresa a los accionistas. Cuando el grado de protección a los inversionistas en la economía es el adecuado y el mercado de capitales se encuentra suficientemente desarrollado la corporación tiende a presentar una estructura dispersa de la propiedad y el control. Esto implica que los accionistas deben delegar su mandato en un grupo de ejecutivos que, en principio, han de velar por los intereses de los accionistas. Debido a la falta de incentivos que tiene el accionista atomizado para monitorear el trabajo de sus agentes el desarrollo de esta estructura de propiedad dependerá, en parte, de que se establezcan mecanismos de control del eventual problema de agencia entre accionistas y ejecutivos. Estos mecanismos pueden ser de dos tipos: mecanismos internos, como la existencia de un directorio, la realización de juntas de accionistas o la implementación de mecanismos de incentivos para los ejecutivos, comostock options; o mecanismos externos, como un activo mercado por control o las leyes y regulaciones existentes. Cuando el grado de protección provisto por los mecanismos tradicionales internos y externos de gobierno corporativo no es suficiente, la respuesta de los accionistas es concentrar la propiedad y el control de la corporación. En estas circunstancias el equilibrio entre los diferentes accionistas se rompe y se producen incentivos para que los accionistas controladores tiendan a expropiar a los demás stakeholders. En economías emergentes no es extraño que se alcance un grado de concentración suficientemente elevado como para que incluso los meca-

2 Ver La Porta et al. (1999) para un detallado resumen de la evidencia acerca del efecto del gobierno corporativo sobre el valor de la empresa. Levine y Zervos (1998) muestran evidencia del efecto del desarrollo del mercado de capitales sobre el crecimiento económico.

3 De acuerdo a la teoría de los flujos de caja libres de Jensen (Jensen, 1986).

$4 \quad$ Ver Jensen y Meckling (1976).

5 Demsetz (1983) y Demsetz y Lehn (1985) plantean este argumento evolutivo respecto a la endogeneidad de la estructura de propiedad de las empresas. 
nismos externos, como el mercado por control (hostil), se hagan insuficientes. Bajo esas condiciones, la implementación de nuevas legislaciones y regulaciones que incrementen la protección efectiva a los inversionistas tenderá a mejorar, como se dijo, el desarrollo del mercado de capitales, redundando en un mayor crecimiento económico agregado.

Sin embargo, no sabemos con certeza cuáles formas de incrementar la protección efectiva a los inversionistas son más convenientes en los diferentes escenarios económicos. Aparentemente, diferentes formas de gobierno corporativo tienden a incentivar el desarrollo de distintos sectores de la actividad económica y son más beneficiosas en diferentes etapas del desarrollo de una economía.

El propósito de este artículo es doble. En primer lugar, se busca estructurar un marco conceptual sencillo que permita ordenar la discusión acerca de las diferentes formas de gobierno corporativo y de su efecto sobre la estructura y desempeño de la empresa. Este marco conceptual se construye en torno a cuatro preguntas fundamentales: (i) ¿qué es el gobierno corporativo?; (ii) ¿existe una forma óptima de gobierno corporativo?; (iii) ¿cuál es el efecto de la estructura financiera y corporativa sobre el desempeño económico?; y (iv) ¿cómo se logra una estructura específica de gobierno corporativo? Las respuestas a cada una de estas preguntas se plantean sucesivamente en las secciones 2 a la 5 con la ayuda de la literatura teórica y empírica relevante. Implícita en la estructura de este artículo y en su desarrollo se encuentra la hipótesis de la selección natural de la estructura de gobierno corporativo, en el sentido que en el largo plazo sobreviven las estructuras que tienden a mejorar el desempeño de las empresas. ${ }^{6}$ El artículo, sin embargo, discute la validez de esta hipótesis y la contrasta con otras hipótesis alternativas tanto en teoría como a la luz de la evidencia empírica. En particular, se discute el significativo rol de los sistemas legales y regulatorios en condicionar el desarrollo de las estructuras de gobierno corporativo en el mundo. En el marco de esta discusión, este artículo enfatiza aquellos aspectos que pueden ayudar a entender mejor el funcionamiento y efecto del gobierno corporativo en el caso de empresas que funcionan en economías emergentes. También se discute el tipo de factores exógenos, relacionados con regulaciones, que pueden ayudar a mejorar las prácticas de gobierno corporativo en una economía emergente como la chilena.

El segundo objetivo que se plantea este artículo es describir la estructura de gobierno corporativo de las sociedades anónimas abiertas chilenas. Para ello, en la sección 6 se revisa y discute el grueso de la incipiente literatura académica sobre gobierno y estructura corporativa en Chile, a la luz del marco conceptual desarrollado previamente.

Debido a lo anterior, este artículo no constituye, en estricto rigor, una revisión bibliográfica sobre el gobierno corporativo. Más completas revisiones de la literatura sobre gobierno corporativo se pueden encontrar en Shleifer y Vishny (1997), Caprasse y Setareh (1998). 


\section{2. ¿Qué es el Gobierno Corporativo?}

La idea de un gobierno se relaciona con el ejercicio de la autoridad en la toma de decisiones que afectan a los intereses de distintas partes. Sin embargo, la teoría micro-económica clásica de la firma no hace referencia explícita a la necesidad de definir los procedimientos mediante los cuales se toman decisiones al interior de la empresa. ¿Por qué debería ser necesario establecer procedimientos cuando la competencia de mercado es capaz de asignar eficientemente los recursos en la economía?, ¿o no lo es?

Zingales (1997) plantea que en muchas transacciones económicas "no estandarizadas" la mano invisible de Adam Smith no es capaz de asignar eficientemente los recursos sin el uso de alguna forma de autoridad. La razón es que en estas transacciones: (i) se generan cuasi-rentas; y (ii) las cuasi-rentas no pueden ser asignadas en una forma obvia ex-ante. Las cuasi-rentas se producen debido a la existencia de complementariedad y externalidades en los diferentes aportes de quienes participan en una relación económica que hacen que el valor del todo (la empresa) sea distinto a la suma de sus partes. Las cuasi-rentas no pueden ser repartidas ex-ante debido a que no es posible confeccionar contratos perfectos que contemplen todas y cada una de las eventualidades que puede sufrir la relación económica establecida, o a que el cumplimiento de dichos contratos no puede ser garantizado debido a un pobre sistema judicial.

En otras palabras, cuando la relación económica o empresa genera rentas que no pueden ser distribuidas a priori, el derecho a tomar decisiones ex-post es valioso puesto que aumenta el poder negociador para capturar dichas rentas. A este derecho se le conoce como el control residual y en las corporaciones modernas recae, en principio, sobre los accionistas.

El ámbito del gobierno corporativo depende, por otra parte, de la precisa definición de empresa o corporación que se tenga. Una definición actual de la empresa se debe a Grossman y Hart (1986). Ellos definen a una empresa como una colección de activos físicos bajo un contrato de propiedad común. Esta definición enfatiza como aspecto fundamental de la corporación a la propiedad común y, por lo tanto, a los incentivos tipo free-rider que se producen bajo este régimen de propiedad. Es decir, cuando existen muchos dueños de un mismo set de activos ninguno tiene el incentivo suficiente para invertir recursos en administrar o monitorear la administración de esos activos. Bajo esta situación la empresa debe manejarse a través de representantes o agentes de los dueños, y en presencia de información imperfecta, se tiene el tradicional problema de agencia entre los accionistas y los ejecutivos de una empresa.

Según Zingales (1997) esta definición tiene el problema de olvidarse de los proveedores de otros activos e inversiones que son también importantes para la empresa. Así, se podría plantear alternativamente que una empresa es un conjunto de inversiones más o menos específicas en capital físico, capital humano, trabajo, relaciones con proveedores, preferencias de los clientes etc., que no pueden ser replicadas en forma obvia por el mercado. En esta relación todos contribuyen a la empresa y no únicamente los accionistas. En la literatura académica se conoce a 
los contribuyentes con inversiones específicas a la empresa comostakeholders, $\mathrm{y}$ en principio todos ellos desean obtener un retorno razonable por sus inversiones. La importancia, entonces, de un adecuado gobierno corporativo radica en ofrecer un conjunto de condiciones que garanticen a los diferentes stakeholders que podrán recuperar su inversión, más alguna remuneración por ella. Esta visión cuestiona, por lo tanto, la lógica de que todo el derecho al control residual de la corporación recaiga sobre los accionistas.

Está claro, entonces, que un adecuado gobierno corporativo importa desde el punto de vista de la distribución de rentas. Sin embargo, el gobierno corporativo tiene, además, un efecto importante sobre la asignación eficiente de recursos. En primer lugar, dependiendo de la estructura de gobierno corporativo los participantes en la empresa pueden decidir invertir una cantidad sub-óptima de recursos. Por ejemplo, un empleado puede invertir menos que el óptimo en capital humano específico a la empresa si no considera que esa inversión será adecuadamente recompensada. Por supuesto, cuanto menos específica sea la inversión realizada mayor será la capacidad del empleado de conseguir una adecuada remuneración en el mercado. En segundo lugar, los agentes invertirán más de la cuenta en actividades que aumenten su capacidad negociadora ex-post. Por ejemplo, un gerente puede sobre especializar a la empresa en un rubro en el que él es más experto con el fin de hacerse relativamente más indispensable para la empresa (Shleifer y Vishny, 1986). El efecto del gobierno corporativo sobre el desarrollo del mercado de capitales y sobre el desempeño económico se discute más adelante en este artículo.

\section{3. ¿Existe una Forma Óptima de Gobierno Corporativo?}

Una vez definido lo que entendemos por una estructura de gobierno corporativo, es necesario plantearse cuál es la mejor forma de gobierno corporativo, si es que existe, y qué políticas y variables son importantes para alcanzarla. En esta sección se discute el efecto de una determinada estructura de gobierno corporativo sobre el desempeño económico. El efecto de los sistemas legales y regulatorios sobre la estructura de gobierno corporativo se discute en la siguiente sección.

\section{i. $\quad$ Rol de los accionistas}

En principio, se podría argumentar que todos los diferentes stakeholders de una corporación tienen la misma importancia. Es decir, desde el punto de vista de la asignación eficiente de recursos, el gobierno corporativo debe proveer de garantías contra la potencial expropiación de sus inversiones en la empresa a todos ellos. En la práctica, sin embargo, la corporación moderna parece privilegiar a una clase particular de inversionistas: los accionistas. Ellos son quienes retienen de hecho el control residual sobre los activos de la empresa. El éxito de la corporación moderna en economías con mercados de capitales desarrollados como 
los Estados Unidos e Inglaterra parece indicar que debe haber alguna razón que justifique que los accionistas tengan el derecho residual sobre el control de la corporación. Una posible explicación de esta asignación se obtiene combinando varios argumentos. En primer lugar, se sostiene que los otros stakeholders de la corporación tienen mejor capacidad de negociación ex-post sobre las cuasi rentas generadas por la empresa, porque normalmente la propiedad del activo productivo con el que ellos contribuyen a la corporación permanece en su poder (Williamson, 1985). Este es el caso, por ejemplo, de oferentes de capital humano que pueden dejar la firma cuando quieran y llevarse el capital consigo.

En segundo lugar, Rajan y Zingales (1998) argumentan que los propietarios de activos en relaciones económicas bajo contratos incompletos tenderán a subespecializar sus activos para evitar ser expropiados en el futuro. Bajo este punto de vista será óptimo que los proveedores de fondos entreguen sus activos a la corporación, retengan el control residual sobre ellos y no participen directamente en la decisión de cuánto especializarlos. Es decir, el control residual se le debe entregar a los accionistas para compensarlos por el hecho de que su capital va a ser especializado para el beneficio de la firma.

En tercer lugar, Fama y Jensen (1998) sostienen que los accionistas de una corporación tienen la ventaja respecto a otros stakeholders de una mejor capacidad de diversificar el riesgo generado por la propiedad de activos específicos a la corporación y, por lo tanto, deberían cobrar menos por asumir ese riesgo que, por ejemplo, los proveedores de capital humano. Este razonamiento implica que es mejor asignar el derecho sobre los flujos de caja residuales a los accionistas. Por otro lado, Fama y Jensen arguyen que los accionistas "garantizan" a los otros stakeholders que no serán expropiados entregando parte de su riqueza a la empresa para que sea invertida en activos específicos.

Los tres argumentos anteriores implican no sólo que el derecho residual de control debería recaer sobre los accionistas, sino que además, es importante que éstos deleguen en otros agentes la decisión respecto a cuánto especializar la inversión en la empresa, y sean capaces de transar sus derechos sobre la empresa en un mercado lo más competitivo posible. Este último aspecto es fundamental para lograr que el horizonte relevante para el accionista a la hora de evaluar la política de inversiones de la empresa sea equivalente al horizonte que tiene la corporación. Esta equivalencia de horizontes se consigue cuando el precio de mercado de las acciones de la empresa refleja efectivamente el valor presente de las oportunidades de inversión futuras de la empresa. Es decir, en un mercado de capitales suficientemente desarrollado y competitivo.

\section{ii. La Corporación Berle y Means}

La discusión anterior plantea una consecuencia fundamental para la estructura recomendada a la corporación. Se vio que es óptimo que los accionistas deleguen la decisión de especialización de las inversiones en otros agentes y que, además, deberían diversificar el riesgo asumido mediante un portafolio construido para ese fin. Por lo tanto, la estructura óptima para una corporación que opera en 
un mercado de capitales competitivo consistiría en una gran cantidad de accionistas pequeños, propietarios de acciones comunes, que no tengan otro rol en la empresa y que deleguen su autoridad en un directorio y un grupo de ejecutivos.

A la estructura de empresa descrita en el párrafo anterior se la considera un paradigma tradicional desde Berle y Means (1932) en donde los accionistas se encuentran expuestos a ser expropiados por sus agentes. Hay tres formas básicas mediante las que los accionistas de este tipo de corporaciones se protegen de la expropiación o costo de agencia. En primer lugar, los inversionistas requieren de protección legal en la forma de leyes y regulaciones que protejan los derechos de los accionistas y obliguen a los ejecutivos a informar veraz y oportunamente al mercado. En la próxima sección se discuten estos aspectos en más detalle.

En segundo lugar, los accionistas de la empresa de propiedad difusa pueden ser protegidos mediante el desarrollo de un mercado activo por el control corporativo. Este mercado funcionaría como una "espada de Damocles" sobre los ejecutivos, que en caso de descuidar sus deberes y reducir el valor de la empresa podrían ser reemplazados por alguien que estuviera dispuesto a comprar su entrada a la compañía. Este mercado se desarrolló en Estados Unidos a partir del surgimiento del mercado de deuda corporativa chatarra, que facilita el financiamiento de este tipo de operaciones.

En tercer lugar, los accionistas pueden protegerse a sí mismos simplemente implementando incentivos a sus agentes en la forma de bonos, remuneraciones amarradas a desempeño, stock options o despido condicionado.

A pesar de que el modelo de corporación con propiedad dispersa descrito anteriormente debería prevalecer en economías que tienen mercados de capitales desarrollados y adecuada protección legal para sus inversionistas, esto no es así en la práctica. Por un lado, Demsetz y Lehn (1985) encontraron una fuerte dispersión en la estructura de propiedad de las corporaciones norteamericanas. Este resultado indicaría que, en el mercado de capitales más desarrollado, coexisten diferentes empresas resolviendo el problema de agencia de formas diversas, o incluso aceptando diferentes grados de costo de agencia. La interpretación de este resultado es que si estructuras más desconcentradas soportan mayores costos de agencia es porque debe haber mayores beneficios de otro tipo asociados a esta estructura. En particular, se ha sugerido que el costo de capital de la firma disminuye con la dispersión de la propiedad debido a la mayor liquidez para sus instrumentos de financiamiento y los mayores beneficios de diversificación de portafolio que pueden lograr sus inversionistas. ${ }^{7}$

Por otro lado, Franks y Mayer (1998) encuentran que sólo Estados Unidos e Inglaterra presentan un porcentaje importante de sus empresas listadas en alguna bolsa formal y, además, un grado relativo de dispersión de propiedad. La diferencia principal es que mientras que en Estados Unidos el mayor accionista suele ser un individuo, en Inglaterra es un inversionista institucional. Por otro lado, los demás países europeos tienen relativamente pocas empresas listadas en 
bolsa, y en el caso de Francia y Alemania que son los casos con mercados de capital más desarrollados, la mayoría de las empresas tiene al menos un accionista con sobre el $25 \%$ de las acciones de la empresa y, en muchos casos, un accionista ejerce el control indiscutible de la empresa.

No sólo hay diferencias en el grado de concentración de la propiedad entre las empresas de diferentes economías europeas, sino que existen marcadas diferencias en el tipo de inversionista que ejerce un rol protagónico en la empresa. En este sentido el modelo alemán ha sido un paradigma de la importancia de un sector bancario activo en el desarrollo industrial y corporativo. Kennedy (1987) destaca que el sector bancario alemán es responsable de suministrar fondos en gran escala para el desarrollo del sector industrial en Alemania, y que este proceso explica el mayor crecimiento y mejor desempeño económico de Alemania respecto a Inglaterra en el pasado. El modelo alemán consiste en términos generales en un sector bancario fuerte comprometido en el financiamiento de empresas en el sector industrial, y con participación directa en la propiedad de estas empresas y en su directorio.

El extraordinario éxito en un principio del modelo japonés de desarrollo se asoció también al efecto positivo que los bancos tienen al participar en la estructura corporativa. Hoshi, Kashyap y Scharfstein (1990) encontraron un efecto positivo en el desempeño de empresas japonesas que tenían una relación estrecha con un banco al pertenecer al mismo conglomerado industrial y financiero.

En el último tiempo, sin embargo, la popularidad del modelo de desarrollo corporativo apoyado en un sistema bancario fuerte ha perdido terreno. En primer lugar, ha contribuido a este cambio de opinión la constatación de que el "milagro alemán" no era tal y que no se aprecia un efecto positivo de la mayor concentración de propiedad y especialmente del rol protagónico de los bancos alemanes en el desempeño de sus empresas (Franks y Mayer, 1998). En segundo lugar, el pobre desempeño reciente de la economía japonesa y la crisis asiática han llamado la atención sobre la, muchas veces, pésima asignación de recursos de inversión que se realiza en economías donde existen fuertes vínculos de propiedad entre bancos y corporaciones, y donde no se realiza una fuerte supervisión de estas relaciones.

Una desviación del modelo clásico de la corporación Berle y Means que se observa en muchas economías combina propiedad relativamente dispersa con la presencia de uno o varios inversionistas institucionales, generalmente administradores de fondos de pensiones. En estas empresas, se considera que los inversionistas institucionales ejercen un rol protagónico en el monitoreo del comportamiento de los ejecutivos generando una externalidad positiva hacia los accionistas minoritarios (Black, 1992, y Starks, 2000). Sin embargo, también se ha sostenido que el rol como monitores de los inversionistas institucionales se ha exagerado, puesto que estos inversionistas están más preocupados de la liquidez de sus inversiones que de que se maximice el valor de las empresas en las que invierten. Este conflicto de intereses disminuiría el efecto benéfico de su participación en las corporaciones (Coffee, 1991).

En definitiva, la discusión acerca de la estructura óptima de gobierno corporativo ha dejado de centrarse en la elección entre un sistema basado en los 
bancos versus uno basado en el mercado de capitales, y se ha movido hacia el tema de la adecuada protección a los inversionistas cualquiera sea el tipo. Estos enfoques se discuten más adelante en este artículo.

\section{iii. Conglomerados en economías emergentes}

La evidencia muestra que no se observa la existencia de la empresa Berle y Means a no ser que exista un adecuado grado de protección tanto a accionistas como a otros inversionistas, y que el mercado de capitales se encuentre desarrollado (La Porta et al., 1999). La forma dominante de empresa en el resto del mundo es la de estructuras con propiedad concentrada. Esta evidencia, en principio, es consistente con la hipótesis sostenida por La Porta, López-de-Silanes, Shleifer y Vishny (LLSV) en el sentido que el principal determinante de la estructura y gobierno corporativo es el grado de protección legal a los inversionistas. Esto es así, en parte, porque en la mayoría de las economías del mundo no existe un mercado de capitales suficientemente desarrollado como para generar un mercado por control activo y eficaz. Además, los incentivos a ejecutivos no son empíricamente importantes, y en muchos casos podrían, de hecho, usarse como un mecanismo adicional de expropiación (Jensen y Murphy, 1990).

En economías con baja protección legal a los inversionistas, la mejor forma de protegerse parece ser eliminando el problema de free-rider aumentando la concentración en la propiedad de la empresa y generando algún tipo de accionista controlador, ya sea otra empresa, una familia, un banco o el Estado. Esta solución de gobierno corporativo no está exenta de problemas. La existencia de un accionista controlador implica que los accionistas minoritarios así como otros inversionistas y stakeholders se encuentran expuestos a ser expropiados. La idea es que el controlador, a diferencia del empresario, financia sólo un porcentaje de los costos asociados a la provisión de beneficios privados del control y, por lo tanto, puede ser óptimo para él desviarse de la política óptima desde el punto de vista del valor de la empresa para su propio beneficio (Jensen y Meckling, 1976). Cuando este efecto es importante y generalizado, los inversionistas minoritarios se retiran del mercado de capitales y se hace más difícil para las empresas conseguir financiamiento.

De hecho, la figura más común en economías emergentes y en desarrollo es el conglomerado o grupo. Esto es un grupo de empresas dedicadas a negocios no necesariamente relacionados, pero que tienen un controlador o accionista mayoritario común o que se encuentra relacionado mediante pactos o sociedades. Estos conglomerados ejercen control sobre las empresas afiliadas mediante estructuras piramidales, series accionarias preferentes y tenencias cruzadas de acciones. El objetivo normalmente es aumentar los derechos políticos o de voto en la corporación en mayor proporción que los derechos sobre los flujos de caja o dividendos (La Porta et al., 1999; Wolfenzon, 1999; Bebchuck, Kraakman y Triantis, 1999). Estas estructuras corporativas complejas significan un aumento en los incentivos que tienen los controladores para expropiar a los accionistas minoritarios. Además, mediante estos mecanismos los controladores construyen un escudo que 
evita las tomas hostiles de control eliminando una de las formas naturales de gobierno corporativo y control externo del problema de agencia entre accionistas controladores y minoritarios.

Además de los problemas de expropiación a los accionistas minoritarios se ha argumentado que las estructuras tipo conglomerado generan otros costos económicos. Así, por ejemplo, en un conglomerado, el sistema de incentivos utilizado para alinear los objetivos de los dueños y los administradores es complejo dado el mayor número de empresas y administradores, por lo que los problemas de agencia tradicionales podrían exacerbarse, a no ser que el conglomerado sea manejado directamente por sus dueños (McConnell y Servaes, 1990). Por otro lado, el conglomerado puede producir problemas de mala asignación de capital, por una tendencia a la sobre diversificación, dado la dificultad de generar conocimiento especializado en muchas áreas simultáneamente (Khanna y Palepu, 1998a).

A pesar de los problemas que se suscitan cuando las corporaciones toman la forma de conglomerados, el uso generalizado de estas estructuras en economías en desarrollo y emergentes y su persistencia en el tiempo indican que esta forma de organización presenta algún tipo de beneficio comparativo respecto a otras estructuras corporativas (Lefort y Walker, 2000a; Khanna y Palepu, 1998a). Las principales razones que se mencionan para explicar que la afiliación a un conglomerado puede ser beneficiosa en una economía emergente se relacionan con la existencia de problemas de mercado causados por información imperfecta, mala regulación y supervisión, corrupción, mercados de capitales e intermediarios financieros poco desarrollados y poco competitivos.

Los beneficios de la afiliación se deben entonces a la creación de sinergias operacionales, administrativas y financieras entre las empresas pertenecientes al conglomerado. Las falencias del mercado de capitales, en general, y la falta de intermediarios financieros competitivos es contrarrestada mediante la creación de un mercado de capitales interno, que permite ahorrar en costos de transacción y asignar fondos entre los diferentes negocios del conglomerado. Los conglomerados en economías emergentes atesoran poder político que les permite sortear la burocracia estatal y la corrupción. Además, cuando los activos del grupo alcanzan un tamaño no trivial comparado con la economía local y no existe un mercado de capitales líquido y profundo donde diversificar riesgos de portafolio, la organización en la forma de un conglomerado puede constituir una adecuada estrategia de diversificación del riesgo de la compañía que también ha sido considerada un beneficio (Claessens, Djankov y Klapper (1999)).

\section{iv. Mercado por control}

En economías con mercados de capitales desarrollados y baja concentración de la propiedad, el mercado por control es uno de los principales mecanismos externos de control corporativo. Normalmente, las tomas de control se realizan bajo dos modalidades. En la primera, conocida como mecanismo de mercado, la transacción se realiza privadamente y los accionistas minoritarios no tienen ninguna participación. En la segunda modalidad, el comprador realiza una oferta pública 
de acciones (OPA) por un porcentaje determinado de las acciones de la empresa, que se asume le permite tomar el control. En caso de que más acciones que las deseadas se tiendan, éstas se adquieren a prorrata entre los accionistas que quieran vender. La diferencia entre los dos mecanismos de compra es que en la OPA los accionistas minoritarios ganan la opción a participar en la transacción bajo las mismas condiciones que el controlador. Intuitivamente, bajo el mecanismo de la OPA, el controlador actual debe renunciar a cualquier ventaja que hubiera tenido sobre los minoritarios como consecuencia de sus beneficios privados. Por esta misma razón, en aquellos casos en los que el controlador pueda bloquear la realización de una toma hostil, la exigencia de OPA puede impedir que cambios en el control de la empresa que podrían ser deseables desde un punto de vista social no se realicen (Lefort y Walker, 2001).

La evidencia para el caso de empresas norteamericanas indica que la principal ganancia que puede hacerse en una toma de control está generalmente asociada a la potencial disminución del problema de agencia entre accionistas y gerentes, y no necesariamente a la posibilidad de impedir expropiación a gran escala. Los estudios que miden el premio pagado por el mercado por acciones con derechos superiores de voto indican que este premio por control se encuentra entre 3 y $5 \%$ al considerar muestras grandes de acciones con alta liquidez en Estados Unidos. Este valor depende de la probabilidad de ocurrencia de una lucha por el control de la corporación, y del sobreprecio que esperan recibir las acciones con derechos preferentes de voto en ese caso (Barclay and Holderness, 1989, y Zingales, 1995). Sin embargo, esta evidencia no es generalizable. En el caso de países con mercados de capital menos desarrollados como Italia, por ejemplo, la evidencia indica que los premios por control alcanzan al 80\% (Zingales, 1994).

Respecto al efecto que las transacciones que involucran cambios en el control tienen sobre el valor de las acciones comunes, existen diversos estudios que analizan diferentes tipos de fusiones y adquisiciones. En particular, Holderness y Sheehan (1988) estudian este efecto en una muestra de empresas norteamericanas que poseen un accionista controlador que tiene un porcentaje tal de las acciones que es suficiente para impedir tomas de control hostiles. Esta evidencia indica que las acciones comunes de empresas de este tipo que sufren un cambio en el controlador, cuando no se produce OPA simultáneamente, aumentan de precio en aproximadamente $10 \%$. En cambio, en aquellas transacciones donde se realiza una OPA, el precio de la acción común termina aumentando en un $20 \%$. Esta evidencia es consistente con la idea de que la OPA ayuda a seleccionar transacciones en las que el valor de los flujos de caja disponibles para los accionistas serán mayores. 


\section{4. ¿Cuál es el efecto de la Estructura Financiera y Corporativa sobre el Desempeño Económico?}

\section{i. Desarrollo financiero y crecimiento económico}

Desde hace ya un tiempo se conoce la relación positiva que existe entre el desarrollo financiero de una economía y su crecimiento económico. King y Levine (1993) encontraron que el volumen de crédito, especialmente al sector privado, aumenta significativamente la tasa de crecimiento de los países. Por otro lado, Levine y Zervos (1998) muestran empíricamente que la mayor liquidez del mercado de capitales accionario y el mayor desarrollo del sector bancario afectan positivamente al crecimiento económico. De acuerdo a Beck, Levine y Loayza (2000) hay tres canales mediante los que este efecto se transmite principalmente. En primer lugar, mayor desarrollo financiero puede fomentar mayor ahorro. En segundo lugar, el sector financiero ayuda a canalizar estos ahorros en la forma de fondos externos disponibles para las empresas y aumenta la acumulación de capital. Finalmente, un mayor número y competitividad de los intermediarios financieros mejora la asignación de los fondos disponibles y aumenta el crecimiento económico. El efecto positivo del mayor desarrollo financiero se produce, de hecho, más fuertemente en empresas que pertenecen a sectores que presentan una escasez relativa de financiamiento externo (Rajan y Zingales, 1998).

A pesar del relativo consenso que existe respecto a la relación entre desarrollo financiero y crecimiento económico, no parece haberse alcanzado un consenso similar en la relación entre un tipo específico de sistema financiero y el desempeño económico de las empresas que lo adoptan. Es decir, no sabemos si una forma particular de desarrollo financiero es mejor que otra. Por ejemplo, no estaría del todo claro si conviene privilegiar el desarrollo de un sistema bancario fuerte siguiendo al modelo alemán, o enfatizar el desarrollo de un mercado de capitales accionario siguiendo el modelo norteamericano (Carlin y Mayer, 2000). ¿Producen los dos tipos de desarrollo financiero el mismo resultado?

Intuitivamente, se podría esperar que diferentes formas de desarrollo de los mercados financieros afecten en forma distinta a diferentes clases de empresas. La idea es que la existencia de economías de escala en el monitoreo hace particularmente eficiente a los bancos a la hora de vigilar a las corporaciones en comparación con inversionistas individuales. Por otro lado, el mercado de capitales presenta ventajas al agregar información dispersa que se encuentra en las manos de muchos pequeños inversionistas.

Además, se puede pensar que las inversiones que se benefician más relativamente de un adecuado monitoreo son aquellas que se hacen en actividades tradicionales, donde la principal fuente de incertidumbre es la calidad del acreedor y de su gestión. En cambio, la capacidad de agregar información dispersa es más útil en el caso de actividades que involucren el uso de nuevas tecnologías. En esos casos lo importante es evaluar el valor esperado de los proyectos a partir de muy diversas percepciones respecto al resultado final. Lo anterior no significa 
que la calidad del acreedor y gestor no importe, sino que existe mucha más incertidumbre relativamente respecto a la calidad del proyecto.

Consistentemente con lo anterior, se ha encontrado que las economías con alto grado de desarrollo de su mercado de capitales presentan un alto crecimiento del financiamiento externo accionario y de las industrias intensivas en nuevos conocimientos. Este efecto se produce principalmente a través de la inversión en investigación y desarrollo. Por el contrario, en economías con muy alto volumen de crédito bancario y con participación de los bancos en el gobierno corporativo se observa mayor crecimiento en industrias que requieren inversiones de largo plazo con tecnologías basadas fuertemente en imitación. Esto es especialmente cierto en el caso de economías con un sector bancario concentrado en unos pocos bancos grandes debido a la facilidad que, en teoría, estos tienen para refinanciar proyectos de gran escala (Carlin y Mayer, 1999).

\section{ii. $\quad$ Gobierno corporativo y desempeño económico de la empresa}

En resumen, existe consenso respecto a que un mayor desarrollo del sistema financiero mejora las posibilidades de crecimiento de una economía. Sin embargo, también parece ser cierto que el tipo de desarrollo financiero que se produce condiciona los sectores que van a verse más beneficiados por este desarrollo y, por lo tanto, diferentes tipos de mercado financiero pueden ser más convenientes en diferentes etapas de desarrollo de una economía. Algo similar ocurre cuando consideramos el efecto de diferentes estructuras de gobierno corporativo sobre el desempeño económico de la empresa. Nuevamente, la idea es que no está claro que exista una estructura que domine a las otras en todas las situaciones.

No obstante lo anterior, hay que tener presente que el mecanismo principal mediante el cual una adecuada estructura de gobierno corporativo mejora el desempeño económico agregado es a través de su efecto sobre el desarrollo del mercado de capitales. Cuando la estructura de gobierno corporativo protege efectivamente los intereses de los inversionistas externos a la empresa, éstos estarán dispuestos a pagar más por las acciones y bonos corporativos, incentivando a las empresas a emitir estos instrumentos para financiar nuevos proyectos (La Porta, López-de-Silanes, Shleifer y Vishny, 2000). Así, por ejemplo, La Porta, López-deSilanes, Shleifer y Vishny (1997) encuentran que en aquellos países que presentan un alto grado de protección a los accionistas se observa mercados accionarios con mayor valor de mercado, más empresas transadas y mayor frecuencia de ofertas iniciales de acciones. Por otro lado, La Porta, López-de-Silanes, Shleifer y Vishny (1999) encuentran que las empresas de países con mejor protección a los inversionistas tienen un mayor q de Tobin. ${ }^{8} 9$ clásico de arbitraje. Si el valor de mercado del capital de una empresa excede su costo de reposición en el mercado, entonces la firma está creando valor y podría incrementarlo en mayor medida invirtiendo unidades adicionales. En el desarrollo de la teoría de inversión, Hayashi (1982) distingue entre el q promedio y el marginal y establece que en presencia de costos de ajuste convexos las decisiones de inversión se toman en el margen. 
A pesar del efecto positivo que, en términos generales, induce la mejor protección a los accionistas, la forma precisa de esta protección condicionará el tipo de inversionista más favorecido, el tipo de financiamiento obtenido y la clase de empresas y actividad económica que se desarrolle. Por otro lado, la forma de estructura corporativa que prevalezca en una economía dependerá de factores institucionales como leyes, regulaciones, eficiencia de la supervisión y del sistema judicial, entre otras. Un aspecto fundamental que se infiere de la evidencia empírica existente es que la corporación Berle y Means de propiedad atomizada es una excepción a la regla en la mayoría de los países. De hecho, sólo se observa en economías con excelente protección a los inversionistas y donde los bancos y los inversionistas institucionales no son demasiado importantes individualmente en relación al tamaño del sector financiero y del mercado de capitales. Por otro lado, en economías con adecuado grado de protección a los inversionistas, pero con inversionistas institucionales o bancos grandes en relación al tamaño de las empresas, y que no enfrentan restricciones respecto a sus inversiones en acciones, lo normal es observar la existencia de un número relativamente reducido de inversionistas de tamaño grande. Finalmente, en economías en desarrollo con baja protección a los inversionistas y mercados de capitales poco desarrollados, se tiene un alto grado de concentración de la propiedad con, usualmente, la presencia de algún accionista controlador, y se observa la formación de grupos económicos o conglomerados.

En cada una de estas situaciones la estructura corporativa busca adaptarse al marco institucional en el que se desenvuelve la empresa. Sin embargo, el resultado final no siempre es óptimo para todos los tipos de actividad económica y para la etapa del proceso de desarrollo en que se encuentre el país. De hecho, en economías con baja protección a los accionistas es muy posible que la estructura con inversionista único (empresario dueño) permita proveer financiamiento más barato que una alternativa con múltiples inversionistas. Sin embargo, Huang y Xu (1998) indican que una estructura corporativa con inversionistas múltiples es meun indicador de desempeño y/o valor de la empresa. La idea es que empresas con alto $\mathrm{q}$ de Tobin indican una mayor capacidad para crear valor $\mathrm{y}$, por lo tanto, mejor desempeño. A pesar de su uso extendido, la q de Tobin, como indicador de desempeño, no ha estado exenta de críticas. Estas críticas se refieren a dos aspectos. En primer lugar, la medición de la q de Tobin no es un procedimiento sencillo. Por ejemplo, el costo de reposición de los activos de la empresa suele aproximarse usando valores libro, lo que implica que tiende a sobreestimarse la q en el caso de empresas con activos intangibles, o con activos fijos en países con inflación y donde no se aplica corrección monetaria. Lewellen y Badrinath (1996) discuten la forma de medir adecuadamente la q de Tobin.

En segundo lugar, se ha discutido la interpretación de la q de Tobin como medida de desempeño. En particular, se arguye que una q de Tobin alta puede indicar cosas como antigüedad de la empresa, oportunidades no explotadas, costos de ajuste y otros que no necesariamente tienen relación directa con mejor desempeño económico. 
jor para financiar proyectos que requieran altas inversiones en investigación y desarrollo puesto que imponen una restricción presupuestaria "dura" que favorece la terminación temprana de proyectos de baja rentabilidad. En cambio, la estructura corporativa dominada por la figura del empresario no provee de dicha restricción y es, por lo tanto, preferible en etapas más tempranas del desarrollo económico de un país donde el proceso de investigación y desarrollo consiste principalmente en la imitación de procesos existentes. Esta hipótesis ha sido, de hecho, corroborada recientemente (Carlin y Mayer, 1999).

Por otro lado, La Porta, López-de-Silanes, Shleifer y Vishny (1999) muestran que existe una asociación entre el grado de protección a los inversionistas y el grado de concentración de la propiedad. Así, la menor protección a los inversionistas a través de leyes, regulaciones y mecanismos corporativos aceptados como directores profesionales y otros, se ve en la práctica compensada por un aumento en la concentración de la propiedad. Es decir, por un lado, los inversionistas buscan protegerse a sí mismos aumentando su poder de voto en la empresa. Por otro lado, esta evidencia puede ser interpretada en el sentido de que una baja protección a los inversionistas aumenta los beneficios privados del control e induce a los controladores a bloquear las oportunidades de una toma de control hostil aumentando la concentración o separando la propiedad del control de la propiedad de los flujos de caja (Bebchuck, 1999).

El efecto que la concentración de la propiedad tiene sobre el desempeño económico no es trivial. Así, a la idea de Jensen (1986) de que menor concentración de la propiedad puede implicar menor costo de capital y mejor desempeño para la empresa hay que agregarle otras consideraciones. Por ejemplo, la hipótesis de Jensen y Meckling (1976) es que una mayor propiedad accionaria en manos de los ejecutivos (o controladores) de la compañía implica una estructura de incentivos más alineada con los intereses de los otros accionistas. Por lo tanto, los costos de expropiación deberían disminuir a medida que aumenta el porcentaje de propiedad del controlador. A lo anterior, Stulz (1988) añade el efecto que un mayor porcentaje de propiedad en manos del controlador tiene al aumentar el premio pagado por eventuales competidores por el control corporativo. Por otro lado, siguiendo el argumento adaptativo de Demsetz y Lehn (1985), tanto la concentración de la propiedad como el desempeño y valor de la empresa pueden verse afectados por factores exógenos que determinen a ambas variables en forma simultánea. En particular, Morck, Schleifer y Vishny (1988) encuentran que la relación entre mayor concentración de la propiedad y desempeño económico no es lineal. Estos autores encuentran que, aunque en promedio la concentración tiene un efecto negativo sobre el valor la empresa, la concentración tiene un efecto positivo para valores bajos de propiedad gerencial, y que esta relación se reversa para propiedad gerencial entre 5 y $30 \%$.

Ya se mencionó que en el caso de economías emergentes con baja protección a los inversionistas, pobre sistema judicial y escaso desarrollo de los mercados de capitales, la forma más común de corporación se encuentra vinculada a los grupos económicos o conglomerados. La evidencia respecto a esta estructura corporativa no es conclusiva. En primer lugar, los estudios muestran que a mayor 
porcentaje de propiedad del controlador del conglomerado sobre los flujos de caja de las empresas mayor valor económico de éstas. Esto es así en el caso chileno (Lefort y Walker, 2001), y para los países del Este de Asia (Claessens, Djankov, Fan y Lang, 1999). Recientemente, Khanna y Rivkin (2000), analizan empresas en 14 economías emergentes y encuentran que las firmas afiliadas a grupos económicos presentan un mayor q de Tobin en 6 países, menor q de Tobin en 3 países y ninguna diferencia significativa en 5 países.

\section{5. ¿Cómo se Logra una Estructura Específica de Gobierno Corporativo?}

En la sección anterior se vio cuáles son las estructuras corporativas más comunes y su efecto sobre el gobierno corporativo. En esta sección se plantea que no es probable que se den las condiciones para que la estructura de gobierno corporativo de un país se mejore sin la intervención externa de la autoridad, y se discuten los principales elementos que deben considerarse para lograr que una reforma a estas estructuras sea efectiva.

\section{i. Protección legal a los inversionistas}

El Teorema de Coase establece que bajo ciertas condiciones los participantes del mercado organizarán sus transacciones en forma eficiente. Estas condiciones son básicamente que los derechos de propiedad se encuentren definidos con claridad y los costos de confeccionar contratos entre privados y hacer que se cumplan sean despreciables. En una economía con esas características deberíamos observar que cada empresa se organiza en función de los mejores contratos posibles. Como los bienes y servicios que son provistos en las economías de mercado actuales pueden ser producidos por diferentes tipos de organización, el principio de competencia de mercado nos dice que debería sobrevivir aquel tipo de estructura de contratos que es capaz de proveer estos bienes y servicios al menor costo posible.

De hecho, la aplicación del Teorema de Coase al problema de la distribución de cuasi-rentas en una empresa implica que independientemente de la ley y de las regulaciones existentes, los participantes en la empresa serán capaces de estipular mediante contratos la mejor forma de realizar esta distribución y obtener, por consiguiente, un adecuado nivel de financiamiento externo. Por supuesto, la validez de este resultado depende, como vimos, de la eficacia del sistema judicial para hacer cumplir estos contratos. Johnson y Shleifer (2000) plantean tres niveles de posición Coasiana: (i) las leyes no importan; (ii) las leyes importan pero las instituciones se adaptan para permitir el uso eficiente de contratos privados; y (iii) aunque las leyes importan y las instituciones domésticas no son capaces de adaptarse suficientemente rápido, las empresas pueden importar contratos internacionales, mediante la emisión de ADR, por ejemplo, lo que les permite lograr la eficiencia. 
Esta discusión es especialmente relevante a la hora de evaluar la posibilidad de implementar reformas que mejoren la estructura de gobierno corporativo en una economía. En primer lugar, si las condiciones para implementar contratos privados en forma eficiente bajo el Teorema de Coase se dan, entonces los agentes privados deberían ser capaces de definir la estructura corporativa más conveniente en cada caso. Por lo tanto, las regulaciones y reformas legales no deberían ser necesarias ni recomendables, por cuanto el regulador puede equivocarse respecto a cuál es la estructura corporativa más conveniente para una determinada etapa de desarrollo.

Al pensar en la estructura corporativa más adecuada para una etapa de desarrollo específica de un país es necesario diferenciar entre dos niveles de reestructuración corporativa. Por un lado, como ya se discutió anteriormente, es normal que las corporaciones se adapten al entorno institucional en el que se desenvuelven. Así, cuando no existen buenos mecanismos externos de gobierno corporativo como un mercado por control activo o un marco legal adecuado, las corporaciones implementan mecanismos internos que suelen reflejarse en un aumento en la concentración de la propiedad. Si bien estos mecanismos pueden ser apropiados como respuesta al entorno, no necesariamente proporcionan los mejores incentivos para el desarrollo del mercado de capitales y el crecimiento económico. Por otro lado, si partiéramos de una economía con un nivel adecuado de protección a los inversionistas, que no discriminara en favor de ninguna clase específica de inversionista o de estructura corporativa, aún podríamos esperar que diferentes estructuras corporativas y de control favorecieran diferentes actividades en distintas etapas del desarrollo.

El punto crucial es que, bajo el Teorema de Coase, las corporaciones de economías con escasa protección a los inversionistas podrán superar esa limitación a través de contratos privados que permitan establecer mecanismos de gobierno corporativo que aseguren adecuada protección a los inversionistas y sean, al mismo tiempo, los más adecuados para el desarrollo económico del país. La implicancia del Teorema de Coase sería un proceso de "convergencia" natural mediante el cual economías poco desarrolladas en cuanto a sus estructuras de gobierno corporativo y mecanismos de protección a los inversionistas se mueven hacia los patrones que se observan en economías más desarrolladas. Esta es la misma implicancia de la hipótesis de selección natural de Demsetz (1983).

Sin embargo, la evidencia empírica es bastante convincente en mostrar que el sistema legal de un país es una variable determinante en las formas de gobierno corporativo que se desarrollan en él, y que las corporaciones y otras instituciones no son capaces de adaptarse para compensar las limitaciones de las leyes (Johnson y Shleifer, 2000). Así, existe prácticamente consenso respecto a que la protección legal a los inversionistas es menor en países que tengan su origen legal en el derecho romano y, a su vez, las economías con mayor grado de protección legal presentan mercados de capital más desarrollados, usan más financiamiento externo a la firma y son menos vulnerables frente a los shocks externos (La Porta, López-de-Silanes, Shleifer y Vishny, 1999 y Johnson, Boone, Breach y Friedman, 2000). 
Desde el punto de vista del regulador, sin embargo, la pregunta relevante es cuáles son los pasos a seguir para lograr un mejoramiento de la estructura de gobierno corporativo que, además, fomente el desarrollo del mercado de capitales y un mayor crecimiento económico. Es decir, si la convergencia no va a ocurrir en forma natural, qué tipo de acciones pueden tomarse para lograrla. Coffee (1999) plantea dos formas de convergencia. Por un lado, se habla de convergencia legal al proceso mediante el cual se logra mover la estructura de gobierno corporativo hacia un modelo deseado mediante la aplicación de una serie de reformas del sistema legal, regulatorio y judicial. Desafortunadamente, la evidencia indica que para tener éxito se requiere de un cambio mayor en esos frentes y que en muchas oportunidades la oposición local a esos cambios puede hacer imposible su aplicación. Así, por ejemplo, los grupos económicos hablan de expropiación a los empresarios cuando se busca implementar leyes que protejan a los accionistas minoritarios. Por otro lado, las reformas que busquen aumentar el uso de fondos externos para empresas pequeñas son discutidas por las grandes empresas que han logrado resolver el problema del financiamiento (Johnson y Shleifer, 2000).

Dado el monumental esfuerzo que implica lograr esta convergencia legal, Glaeser, Johnson y Shleifer (2001) y Berglof y von Thadden (2000) plantean algunas recomendaciones a la luz de la experiencia internacional. En primer lugar, debe tenerse en cuenta que cualquier reforma debe reconocer que existen diferencias aún entre los sistemas de gobierno corporativo que pueden calificarse de exitosos. En segundo lugar, sostienen que la reforma legal es necesaria en la mayoría de los casos. Como ejemplo citan el caso del Neuer Markt en Alemania, diseñado para nuevas empresas y, cuyo éxito, sostienen, se debe al requerimiento de que en dicho mercado se usen las normas internacionales de transparencia y no las alemanas tradicionales. En tercer lugar, concluyen que una reforma es tan buena como el sistema judicial lo permita. En cuarto lugar y, relacionado con lo anterior, plantean que la regulación y supervisión estatal debe usarse en aquellas áreas donde no pueda confiarse en la capacidad del sistema judicial para hacer que el espíritu de las leyes se cumpla. Para el caso de economías en desarrollo se sostiene que es más importante enfocarse en la protección a los acreedores que a los accionistas minoritarios, y que en la mayoría de los casos se requerirá de algún grado de condicionalidad externa para lograr el éxito de la reforma. En general, la recomendación es concentrarse en implementar una buena ley de quiebra que maximice la eficiencia del proceso de cambio en la propiedad de los activos, y preserve el rol penalizador del proceso y la estructura de prioridades original entre los diferentes acreedores.

Cuando la convergencia legal es muy costosa se ha planteado el enfoque de la convergencia funcional. Esta consiste, en general, en fomentar y acelerar la apertura del mercado de capitales al exterior. La idea es que el uso de ADRs o, por ejemplo, el que una firma local sea adquirida por una extranjera que opera en una economía con adecuada protección va mejorando sostenidamente el grado de protección a los inversionistas locales. A pesar de lo anterior, La Porta, López-deSilanes, Shleifer y Vishny (2000) sostienen que este tipo de convergencia suele ser de muy poco efecto para proteger los derechos de acreedores y otrosstakeholders. 


\section{ii. Las recomendaciones de la $O E C D$}

En mayo de 1999 la OECD aprobó los “principios de la OECD para el gobierno de las sociedades." Estos principios resumen una serie de iniciativas y experiencias de países miembros de esta organización. En particular, la OECD establece una serie de recomendaciones en las áreas de: derechos de los accionistas, tratamiento equitativo a los accionistas, la función de los stakeholders en el gobierno de las sociedades, y el rol del directorio. Algunas de las recomendaciones más importantes pueden usarse y, se han usado de hecho, en la implementación de reformas a las leyes de valores y sociedades anónimas de diverso países, entre ellos Chile.

Hay que destacar, sin embargo, que la OECD reconoce que no existe un modelo único de gobierno corporativo. No obstante lo anterior, se establece que existe un conjunto de elementos comunes a todo buen gobierno corporativo. De entre estos elementos cabe destacar los siguientes. En primer lugar, se reconoce que los accionistas tienen un papel privilegiado respecto a otros stakeholders en cuanto a su derecho sobre el control residual de los activos. En segundo lugar, pero muy relacionado con lo anterior, se establece que los accionistas tienen derechos iguales entre ellos y que debe promoverse toda forma de proporcionarles información suficiente respecto a las decisiones que los ejecutivos o el accionista controlador estén efectuando. Se recomienda también que la adquisición del control corporativo esté reglamentada de forma que sea transparente y se realice en condiciones justas y equitativas. En tercer lugar, se recomienda establecer mecanismos regulatorios que aseguren que la corporación y sus controladores respetarán los derechos e intereses de los otros stakeholders de la compañía, entregándoles en forma oportuna y adecuada la información relevante. Finalmente, se establecen las principales responsabilidades y derechos de los miembros del directorio de la corporación. En este sentido, los directores deben actuar en forma diligente, asegurándose que las decisiones que se tomen afecten a los diferentes accionistas en la misma forma. Además, los directores deben ser capaces de tener una opinión informada e independiente de la que sostengan los ejecutivos de la empresa.

Las recomendaciones de la OECD para el buen gobierno corporativo no han estado exentas de polémica. La principales críticas que se les ha hecho han sido dos. Por un lado, la rigidez de la estructura de gobierno corporativo que surge de estas recomendaciones, que contrariamente a lo que la propia OECD sostiene parece desconocer la posibilidad de que existan diversos tipos de gobierno corporativo utilizables en diferentes contextos económicos e institucionales. En segundo lugar, se critica la excesiva importancia que se le da al accionista en desmedro de otros stakeholders. 


\section{6. ¿Cómo es el Gobierno Corporativo en Chile?}

El interés por la estructura de gobierno corporativo en Chile ha crecido en los últimos años tanto a nivel académico como en el sector empresarial y de gobierno. Por una parte, este interés se ha debido a la ola de transferencias de control en importantes empresas chilenas ocurrido en el último tiempo y que gatilló, a su vez, a la reforma a las leyes de Sociedades Anónimas y de Valores, mejor conocida como Ley de OPA, promulgada en diciembre de 2000. Por otra parte, la literatura académica sobre gobierno corporativo en Chile ha ido creciendo al amparo del desarrollo del marco conceptual y empírico a nivel internacional.

El propósito de esta sección es resumir lo que se conoce respecto a la estructura de gobierno corporativo en Chile, a la luz del marco conceptual presentado en las secciones anteriores, revisando la evidencia reciente mostrada en la literatura académica sobre el gobierno corporativo en Chile. La sección se divide arbitrariamente en varias subsecciones que discuten aspectos como la estructura corporativa y de control de las empresas chilenas, las prácticas de los directorios, el funcionamiento del mercado por control en Chile y las características del marco legal.

En términos generales, la estructura de propiedad y de gobierno corporativo que se observa hoy en Chile se ha desarrollado condicionada por eventos políticos y económicos ocurridos en el pasado y se caracteriza por la presencia de grupos económicos o conglomerados que dominan gran parte de la actividad empresarial nacional. ${ }^{10}$

\section{i. $\quad$ Estructura de propiedad de las corporaciones chilenas}

La estructura de propiedad de las empresas chilenas se caracteriza por la alta concentración de la propiedad y la generalizada presencia de grupos o conglomerados. Agosín y Pastén (2000) y Lefort y Walker (2000c) indican que al considerar todas las empresas listadas en bolsa el mayor accionista es propietario, en promedio, del $46.2 \%$ de las acciones, mientras que los tres y cinco mayores accionistas mantienen, en promedio, el $73.6 \%$ y $88.6 \%$ de la propiedad respectivamente.

De hecho, la concentración de la propiedad en Chile en términos del control de la empresa es aún mayor debido a la presencia de estructuras de propiedad interrelacionada que constituyen los llamados "grupos económicos" o conglomerados. ${ }^{11}$ La existencia de estos grupos implica que los diferentes accionistas reportados en la Ficha Estadística Codificada Uniforme (FECU) por las sociedades anónimas abiertas chilenas pueden corresponder, de hecho, a la misma persona utilizando diferentes vehículos de inversión o a diferentes accionistas actuando se ha desarrollado en respuesta a eventos relacionados con cambios políticos importan- 
en común bajo el paraguas de un grupo. Estos grupos dominan el paisaje corporativo chileno. A partir de una muestra compuesta por todas las sociedades anónimas abiertas chilenas no financieras, Lefort y Walker (2000c) encuentran que entre los alrededor de 50 conglomerados registrados por la Superintendencia de Valores y Seguros (SVS) controlan sobre el 73\% de las sociedades anónimas abiertas y sobre el $90 \%$ de sus activos. La propiedad del capital accionario consolidado de las empresas afiliadas a grupos económicos se divide en un $57 \%$ en manos de los controladores, un $25 \%$ repartido entre fondos de pensiones y ADR, y alrededor de un $15 \%$ repartido entre otros accionistas minoritarios. Aproximadamente la mitad de los grupos económicos que operan en Chile son controlados por capital extranjero. Además, el alto grado de concentración de la propiedad y del control no parece estar disminuyendo, sino que por el contrario, ha ido en aumento durante los 90s. De hecho, empresas que fueron privatizadas dispersando su propiedad hacia fines de los años 80 hoy tienen propiedad concentrada.

Con respecto a la estructura de control, los conglomerados chilenos se caracterizan por un férreo control de sus empresas. En primer lugar, como se mencionó, los controladores poseen alrededor del 57\% del capital accionario consolidado del conglomerado. Además, los conglomerados muestran estructuras piramidales que permiten aumentar la cantidad de activos controlados por peso invertido. Así, los controladores en Chile consiguen 2.3 pesos de financiamiento externo por cada peso aportado por ellos. Sin embargo, estas estructuras son relativamente simples. Por un lado, rara vez se observan estructuras con más de tres niveles de consolidación y el uso de series preferentes de acciones es relativamente escaso observándose en menos del $7 \%$ de las sociedades listadas en Chile. Por otro lado, la legislación chilena prohíbe el uso de tenencias cruzadas de acciones.

\section{ii. Composición y prácticas de los directorios}

Los directorios de las sociedades anónimas tienen la función de administrar la empresa en beneficio de los accionistas independientemente del origen de los votos que recibieron para ser elegidos como directores. Por eso, habitualmente se considera que la presencia de directores independientes, aquellos que no tienen vínculos con los ejecutivos o con los controladores de la empresa, es un elemento importante de un buen gobierno corporativo. Evidencia reciente indica que en Chile solo 55\% de los directores de las sociedades anónimas listadas en bolsa no tiene relación familiar o laboral con los controladores y ejecutivos de la compañía en la que se desempeñan como directores, o en compañías relacionadas con ella. ${ }^{12}$ En el caso particular de los directorios de los conglomerados chilenos, la evidencia indica que los controladores de las empresas afiliadas a un grupo económico parecen abusar de personas relacionadas al conglomerado para llenar

A partir de cuestionarios enviados a las sociedades anónimas abiertas chilenas SpencerStuart y la Escuela de Administración de la Pontificia Universidad Católica de Chile confeccionaron un estudio sobre el funcionamiento de los directorios en Chile. 
los asientos disponibles. Lefort y Walker (2000c) miran a la composición de los directorios de empresas afiliadas a conglomerados en 1998 mostrando que, por un lado, cada director se sienta en 1.6 sillas y, por otro lado, menos del 1 por ciento de los directores de empresas pertenecientes a un grupo se sientan en directorios de empresas de otros grupos. Lo anterior indicaría que los controladores son extremadamente celosos de la información relativa a sus empresas y que el director profesional independiente es una figura poco común en las corporaciones chilenas. En el caso de las empresas más grandes que cuentan con la participación de los fondos de pensiones como accionistas, la presencia de directores elegidos con los votos de las Administradoras de Fondos de Pensiones, AFP puede hacer, como se discutirá más adelante, una importante diferencia. ${ }^{13}$

Un indicador tradicional de la profesionalidad de los directorios es el número de comités que funcionan en forma regular en un directorio. Del estudio de Spencer-Stuart se desprende que, en el caso de las sociedades listadas chilenas, el $71 \%$ de estas empresas no tiene ningún comité que opere regularmente en forma periódica.

\section{iii. Inversionistas institucionales}

Como se mencionó anteriormente, los inversionistas institucionales constituyen un importante accionista minoritario. Lefort y Walker (2000b) sostienen que la relativa simpleza que se observa en las estructuras de los conglomerados chilenos se debe, al menos en parte, a las regulaciones impuestas sobre las administradoras de fondos de pensiones respecto a las características que deben tener las empresas en las que ellas deciden invertir.

La experiencia reciente indica, además, que ésta no es la única contribución al gobierno corporativo en Chile de las AFP. De hecho, en varios casos de adquisiciones de empresas chilenas y de decisiones corporativas, en general, las AFP han sido importantes para llamar la atención de la autoridad económica y la opinión pública respecto a supuestas violaciones a los derechos de los accionistas minoritarios. En cualquier caso, la tendencia reciente que han mostrado las AFP para vender sus acciones en negociaciones que se producen en torno a tomas de control indica que ellas también se preocupan de la liquidez de sus inversiones consistentemente con el planteamiento de Coffee (1991).

\section{iv. El mercado por control en Chile}

La alta concentración de la propiedad que se observa en la mayoría de las empresas chilenas constituye un blindaje para el mercado por control hostil. Como se explicó anteriormente la ausencia de un mercado por control hostil significa que, en principio, el controlador podría estar desviando rentas de la empresa en la forma de beneficios privados y disminuyendo, por lo tanto, el valor de la empresa 
en el mercado, sin que potenciales inversionistas pudieran adquirir el control de la corporación contra la voluntad del actual controlador. Cuando, además, la protección legal a los inversionistas es escasa o su aplicación imperfecta, el valor de la expropiación que los accionistas controladores pueden ejercer sobre los otros inversionistas es potencialmente muy alto. Bajo esas condiciones los controladores de las empresas exigirán grandes sobreprecios, con respecto al valor de mercado de las acciones individuales, para estar dispuestos a entregar el control de las corporaciones. Estos sobreprecios deben, en principio, compensarlos por el valor presente de los beneficios privados del control que están entregando.

De hecho, la experiencia chilena reciente muestra que en la gran mayoría de las transacciones que han involucrado una transferencia del control se han pagado sobreprecios muy altos. ${ }^{14}$ Lefort y Walker (2001) estiman el premio por control observado en Chile a partir del análisis de 12 transacciones que involucraron, en promedio, la transferencia de $40 \%$ de las acciones de la empresas y el cambio en el control de Sociedades Anónimas chilenas inscritas en Bolsa durante 1996, 1997, 1998 y 1999. Se encuentra que el sobreprecio promedio en operaciones privadas alcanza alrededor del 70\%. Esta cifra es similar a la reportada por Lüders y Maturana (1999) y por Parisi, Godoy y Parisi (2001).

Los altos sobreprecios pagados privadamente no implican que, en promedio, estas transacciones hayan sido perjudiciales desde el punto de vista de la empresa transferida. En el mismo estudio se encuentra que como resultado del cambio en controlador, el precio de la acción común aumentó en 5\%. Estos resultados se utilizan para calibrar el valor promedio de los beneficios privados del control, encontrándose que los beneficios privados del control representan aproximadamente el $25 \%$ del valor total de las acciones comunes (que refleja el valor presente de los flujos de caja que podrían obtener los accionistas). Esta evidencia es indicativa del potencial para expropiación de los accionistas minoritarios que existe hoy en las empresas chilenas. Sin embargo, la evidencia que muestra que en Chile se hace relativamente poco uso de estructuras piramidales, y que los controladores mantienen porcentajes importantes de la propiedad de las empresas controladas sugiere que los controladores también valoran en forma importante la obtención de buenos resultados en sus empresas en calidad de accionistas.

\section{v. Conglomerados y desempeño económico de las empresas chilenas}

Son numerosos los estudios que analizan las razones para la conglomeración en Chile, y el efecto que ésta tiene sobre el desempeño económico de las empresas. Paredes y Sánchez (1995), y Tarziján (2000) plantean que los grupos económicos en Chile responden a la necesidad de resolver la poca profundidad de los mercados financieros y de factores que afectan a una economía emergente como la chilena. Paredes y Sánchez (1995) muestran que la identidad y características de los grupos económicos en Chile han ido cambiando en respuesta, proba- 
blemente, al desarrollo de la economía chilena y sus mercados. Consistentemente, al menos en parte, con esta visión de los conglomerados Gálvez y Tybout (1985) muestran que al controlar por tipos de industria, las empresas afiliadas a conglomerados tuvieron un mejor desempeño que las no afiliadas durante la crisis de la deuda a principios de los ochenta. En forma similar, Medina y Valdés (1998) encuentran que, en Chile, la sensibilidad de la inversión de las empresas frente a la disponibilidad de fuentes internas de fondos, es menor para el caso de las empresas afiliadas a grupos económicos.

Una visión menos positiva de la conglomeración en economías emergentes tiene relación con la mayor posibilidad de expropiación a accionistas minoritarios que permiten las estructuras de propiedad de los conglomerados. Lo anterior debido a la mayor posibilidad de separar los derechos de flujo de los derechos de control que estas estructuras permiten. Buscando testear esta hipótesis, Lefort y Walker (2001) miran al efecto que la afiliación a los conglomerados tiene sobre el desempeño de las empresas en Chile. Estos autores encuentran que la pertenencia a un grupo económico reduce significativamente el valor de mercado de la empresa aproximado por la q de Tobin. Esto indicaría que, en promedio, los costos de expropiación a los que está expuesta la empresa afiliada son, en promedio, mayores que los beneficios obtenidos por la existencia de un mercado de capitales interno u otros. Sin embargo, en forma consistente con las predicciones de Jensen y Meckling (1976), el valor de la empresa afiliada aumenta con el porcentaje de flujos de caja que es propiedad de los controladores para un valor dado de derechos de voto. Es decir, cuando los controladores aumentan su porcentaje de propiedad de las acciones del conglomerado consolidado el valor de la empresas afiliadas tiende a aumentar.

Esta evidencia indica que, en el caso de Chile, los costos de expropiación a los accionistas minoritarios sobrepasan a los beneficios que potencialmente entrega el conglomerado, cuando los controladores ejercen dicho control con un porcentaje relativamente bajo de la propiedad accionaria (menos del 46\%). Sin embargo, cuando los controladores aumentan su porcentaje de la propiedad del conglomerado, disminuyendo sus incentivos a expropiar a los accionistas minoritarios, los beneficios de la afiliación a un conglomerado terminan aumentando el valor de la empresa.

\section{vi. $\quad$ Marco legal para la corporación en Chile}

El comportamiento de las corporaciones chilenas y su interacción con el mercado de capitales está normado por un conjunto de leyes contenidas en la Ley de Valores y la Ley de Sociedades Anónimas. El cuerpo central de estas leyes se aprobó en 1981 y fue posteriormente modificado en 1989, 1994 y más recientemente en $2000 .^{15}$ el marco de la reforma de los Gobiernos Corporativos o Ley de OPAs. 
Tres mayores entidades supervisoras, supervisan distintos aspectos de los mercados financieros en Chile: la Superintendencia de Valores y Seguros, la Superintendencia de Bancos e Instituciones Financieras y la Superintendencia de Administradoras de Fondos de Pensiones. El Banco Central también participa activamente en la regulación y la supervisión, especialmente en asuntos relacionados con las transacciones internacionales y participantes en el mercado cambiario.

La Superintendencia de Valores y Seguros (SVS) tiene el principal rol en relación con el gobierno corporativo en Chile. Fue establecida en 1980 como una organización autónoma pública dependiente del Ministerio de Hacienda. Entre sus principales atribuciones se encuentra la emisión de normas para las sociedades anónimas y la fiscalización de las leyes de valores y de las sociedades.

Es importante notar que en Chile la autorregulación en los mercados de capital es prácticamente inexistente. Las principales regulaciones son impuestas y supervisadas por las autoridades correspondientes y no hay requisitos adicionales por parte de las bolsas de valores o prácticas relacionadas con el buen gobierno corporativo auto impuestas por las empresas. Por otro lado, aunque el sistema legal chileno se inspira en el código civil francés, la mayoría de las reformas implementadas a las leyes referentes al mercado de valores y a las sociedades anónimas fueron inspiradas en sus homólogas en los EEUU. No obstante lo anterior, el sistema judicial chileno no parece ser suficientemente ágil y flexible para garantizar a los accionistas minoritarios la aplicación a tiempo y en forma efectiva de las normas que buscan protegerlo.

En el ámbito del gobierno corporativo hay tres aspectos referentes a la legislación chilena que vale la pena destacar. En primer lugar, las transferencias de propiedad accionaria que conduzcan a un cambio de controlador deben hacerse a un "precio de mercado" o mediante una oferta pública de adquisición de acciones que asegure términos similares a todos los accionistas. En segundo lugar, las empresas listadas grandes deben constituir un comité de auditoria formado por mayoría de miembros independientes que tiene, entre otras, la función de aprobar todas las transacciones con partes relacionadas aprobadas por el directorio. Finalmente, a diferencia de la mayoría de los países de la región, Chile no ha instituido todavía el concepto de Código de Mejores Prácticas de Gobierno Corporativo como un requisito para que las empresas listadas informen al mercado de su estándar en cuanto a las recomendaciones internacionales respecto al gobierno corporativo. Es importante notar, sin embargo, que en el caso de las empresas que han emitido ADRs, este tipo de práctica está requerido por la equivalente a la SVS en Estados Unidos.

\section{Conclusiones y Recomendaciones de Política}

En los últimos años se ha producido un surgimiento enorme del interés por entender los mecanismos que componen el gobierno corporativo y sus efectos sobre el desarrollo del mercado de capitales y el crecimiento económico. Detrás de 
este fenómeno se encuentran al menos tres razones. En primer lugar, es indudable la contribución académica que ha permitido comprender los alcances del gobierno corporativo, indicando los incentivos económicos que explican las estructuras corporativas que observamos en la realidad y proporcionando abundante evidencia empírica respecto a sus consecuencias. En segundo lugar, es claro que la globalización e internacionalización de los mercados de capitales y del mundo corporativo han hecho ver que existen diferencias fundamentales entre las estructuras corporativas en las distintas economías. Además, se ha hecho evidente que en el caso especial de las economías en desarrollo es necesario establecer leyes y regulaciones que permitan realizar la transición hacia la corporación globalizada con el mínimo perjuicio para los accionistas minoritarios y otros stakeholders locales, manteniendo el trato equitativo entre ellos y los inversionistas extranjeros. Este proceso es fundamental para un desarrollo racional del mercado de capitales local que provea de fuentes de financiamiento externo a empresas que no cuentan con un mercado de capitales interno que las apoye o que se involucren en actividades que requieren de un cierto grado de innovación tecnológica.

Chile ha iniciado ese proceso a raíz del efecto que algunas adquisiciones de empresas locales han generado sobre la opinión pública y de la iniciativa de la autoridad económica en implementar una legislación que mejore el grado de protección efectiva a los inversionistas. En cualquier caso, este proceso no partió de cero, puesto que la legislación chilena en lo referente a las leyes de sociedades anónimas y de valores ya se había reformado en diversas ocasiones bajo la asesoría de organismos multilaterales, y sigue en muchos aspectos importantes el modelo de las legislaciones de países desarrollados, en particular de los Estados Unidos. Además, las reformas económicas tempranas y, en particular, la reforma de pensiones y la legislación que se deriva de dicha reforma ha logrado que nuestra actividad corporativa sea relativamente transparente y se conduzca bajo prácticas aceptables en comparación con lo que se observa en otras economías en desarrollo.

Sin embargo, es importante considerar que existen grandes diferencias en el ámbito del gobierno corporativo entre Chile y países más desarrollados. En particular, la concentración de la propiedad accionaria en Chile es muy elevada y es muy posible que esta situación persista por mucho tiempo. Por otro lado, la aplicación de la justicia en el caso de situaciones complicadas que involucren potenciales violaciones de los derechos de los accionistas minoritarios es todavía lenta y su resultado incierto. Finalmente, desde 1997, las empresas chilenas han usado en forma preferente la deuda como forma de financiamiento por lo que el tema de la protección de los derechos de los acreedores aparece como un importante aspecto desde el punto de vista de mejorar las condiciones para el acceso al financiamiento para las empresas. En esa dirección se podría pensar en una reforma a la Ley de Quiebras. En la medida que el proceso de quiebras se hace más transparente permite disminuir el riesgo que enfrentan los acreedores sin vulnerar las prioridades entre acreedores y el principio de responsabilidad limitada. Hacer más predecible el proceso de transferencia de la propiedad de los activos durante 
la quiebra podría disminuir el costo de financiamiento y la disponibilidad de fondos para muchas empresas pequeñas y medianas.

\section{REFERENCIAS}

Agosín, M. y E. Pastén (2001), Corporate Governance in Chile. Meeting on Corporate Governance in Developing Countries and Emerging Economics by OECD Development Center and the European Bank for Reconstruction and Development. París, abril.

Barclay y Holderness (1989), "Private Benefits from Control of Public Corporations", Journal of Financial Economics, 25.

Bebchuk, L. (1999), The rent protection theory of corporate ownership and control. Unpublished working paper. Harvard Law School: Cambridge, MA.

Bebchuk, L, R. Kraakman y G. Triantis (1999), "Stock Pyramids, Cross Ownership and Dual Class Equity: The Creation and Agency Cost of Separating Control from Cash Flow Rights", NBER WP N 6951.

Beck, T., Levine, R., Loayza., N. (2000), Finance and the sources of growth. Journal of Financial Economics 58, this issue.

Berglof, E., von Thadden, L. (1999), The changing corporate governance paradigm: implications for transition and developing countries. Unpublished working paper. Stockholm Institute of Transition Economics: Stockholm, Sweden.

Berle, A., and Gardiner M. (1932), The Modern Corporation and Private Property (MacMillan, New York, N.Y.)

Black, B. S. (1992a), Agents Watching Agents: The Promise of Institutional Investor Voice. UCLA Law Review 39, 811-893.

Caprasse, J.N. y Shervin Setareh, (1998), “Corporate Governance Survey”, European Business Literature Review 126.

Carlin W., Mayer C. (2000), International Evidence on Corporate Gobernance: Lessons for Latin America. Revista ABANTE, Vol 2, No 2, octubre.

Claessens S., Djankov S., Fan J. y Lang L. (1999 b), Expropriation of Minority Shareholders in East Asia. World Bank, Febrero.

Claessens S., Djankov S., Fan J., Lang L. (1999 a), The Costs of Group Affiliation: Evidence from East Asia. World Bank, Mimeo.

Claessens S., Djankov S., Klapper L. (1999), The Role and Functioning of Business Groups in East Asia and Chile. Revista ABANTE, Volumen 2, $\mathrm{N}^{\circ} 2$.

Claessens S., Djankov S., Lang L. (1999), The Separation of Ownership and Control in East Asian Corporations. World Bank.

Coffee, J. (1999), The future as history: the prospects for global convergence in corporate governance and its implications. Northwestern Law Review 93, 631-707.

Demsetz, H. (1983), The structure of ownership and the theory of the firm, Journal of Law and Economics 26, 375-390.

Demsetz, H. y K. Lehn (1985), The structure of corporate ownership: Causes and consequences, Journal of Political Economy 93, 1155-1177. 
Fama E, Jensen M. (1983), Separation of ownership and control. Journal of Law and Economics 26, pp 301-325.

Franks, J. y C. Mayer (1994), The ownership and control of German corporations, Unpublished manuscript, London Business School.

Gálvez, J. y James Tybout. (1985), “Microeconomic Adjustment in Chile During 1977-81; The Importance of Being a Group". World Development, Vol. 13, №8.

Gibbs PA. (1993), Determinants of Corporate Restructuring: the relative importance of corporate governance, takeover threat, and free cash flow. Strategic Management Journal, Summer Special Issue 14, pp 51 - 68.

Glaeser, E., Johnson, S., Shleifer, A. (2000), Coase v. the Coasians. Unpublished working paper. Harvard University: Cambridge, MA.

Grossman, S. y O. Hart (1986), The costs and benefits of ownership: A theory of vertical and lateral integration, Journal of Political Economy 94, 691-719.

Hansmann, H. (1988), Ownership of the firm. Journal of Law, Economics and Organization 4 (2), pp $267-305$.

Hayashi, F. (1982), Tobin's marginal and average: A neoclassical interpretation. Econometrica 50(1): 213-224.

Holderness y Sheehan (1988), "The Role of Majority Shareholders in Publicly Held Corporations", Journal of Financial Economics, 20.

Hoshi, T., Kashyap, A., Scharfstein, D. (1991), Corporate structure, liquidity and investment: evidence from Japanese industrial groups. Quaterly Journal of Economics 106, 33-60.

Huang, H. y C. Xu (1999), "Boundary of the Firm, Commitment and R\&D Financing", unpublished manuscript, London School of Economics.

Iglesias-Palau, A. (2000), "Pension Reform and Corporate Governance: Impact in Chile", ABANTE, Studies in Business Management, Vol. $3 \mathrm{~N}^{\circ} 1$, October/April, p. 109-141.

Jensen M., Meckling W. (1976), Theory of the Firm: Managerial behavior, agency cost and ownership structure. Journal of Financial Economics 3, pp305-360.

Jensen, M. (1986), “Agency Cost of Free Cash Flow, Corporate Finance, and Takeovers”, American Economic Review, mayo.

Jensen, M. (1989), Eclipse of the modern corporation. Harvard Business Review 67, 61-74.

Jensen, M. y K. J. Murphy (1990), “Performance Pay and Top Management Incentives”, Journal of Political Economy, abril.

Johnson S., Shleifer A. (1999), Coase and Corporate Governance in Latin America. Revista ABANTE, Vol 2, $\mathrm{N}^{\circ}$ 2, octubre

Johnson, S., Boone, P., Breatch, A., Friedman, E. (2000), Corporate governance in the Asian financial crisis, Journal of Financial Economics 58, this issue.

Kennedy, W. (1987), "Industrial Structure, Capital Markets and the Origins of British Economic Decline, Cambridge. Cambridge University Press.

Khanna T., Palepu K. (1998 a), Is Group Affiliation Profitable in Emerging Markets? An analysis of Diversified Indian Business Groups. Journal of Finance

Khanna T., Palepu K. (1998 b), The Future of Business Groups in Emerging Markets: Long Run Evidence from Chile. Mimeo, Harvard Business School, Working Paper 99-077.

Khanna T., Palepu K. (1999), Policy Shocks, Market Intermediaries, and Corporate Strategy: The Evolution of Business Groups in Chile and India. Journal of Economics and Management Strategy, Massachusetts Institute of Technology, Volume 8, Number 2, Summer 1999, pp. 271-310. 
Khanna T., Rivkin J. (2000), Estimating the Performance Effects of Business Groups in Emerging Markets. Harvard Graduate School of Business and Administration. Working Paper, septiembre 1998.

King, R., Levine, R. /1993), Finance and growth: Schumpeter might be right. Quarterly Journal of Economics 108, 717-738.

La Porta R., Lopez de Silanes F., Shleifer A., Vishny R. (2000), Investor Protection and Corporate Valuation. Harvard University, Mimeo.

La Porta, R., F. López de Silanes, a. Shleifer y R. Vishny (1999), “Corporate Ownership Around the World", Journal of Finance, 54.

La Porta, R., F. López-de-Silanes, A. Shleifer y R. Vishny (1997), "Legal Determinants of External finance", The Journal of Finance, julio.

La Porta, R.; F. López-de-Silanes, A. Shleifer y R. Vishny (1996), "Law and Finance”, NBER WP 5661.

lchian A. A, Demsetz H. (1972), Production, Information Costs, and Economic Organization. American Economic Review 62 (5), pp 777-795.

Lefort F., Walker E. (2000 b), The Effects of Economic Shocks on Corporate Governance Systems in Chile. Revista ABANTE, Vol 2, № 2, octubre.

Lefort F., Walker E. (2000 a), Corporate Governance: Challenges for Latin America. Revista ABANTE, $\mathrm{Vol} 2, \mathrm{~N}^{\circ} 2$, octubre.

Lefort F., Walker E. (2000 c), Ownership and Capital Structure of Chilean Conglomerates: Facts and Hypothesis in Chile. Revista ABANTE, Vol 3, $\mathrm{N}^{\mathrm{o}} 1$, abril.

Lefort F., Walker E. (2001), Economic Performance of Conglomerates: Evidence from Chile. Preliminary Version.

Lefort, F. Y E. Walker (2001 b), "Gobierno Corporativo, Protección a Accionistas Minoritarios y Tomas de Control”, Documentos de Discusión 1, SVS, mayo.

Levine, R., Zervos, S. (1998), Stock markets, banks and economic growth. American Economic Review 88, 537-558.

Lewellen, G. W. y S. G. Badrinath (1997), “On the Measurement of Tobin's Q,” Journal of Financial Economics, 44, 77-122.

Lüders, R. and G. Maturana (1999), Foreign Direct Investment Flow Behavior in Chile During the 1990s: Analytical Description and Policy Implications. Mimeo.

Mathiesen, H. (2002), "Managerial Ownership and Financial Performance," Ph.D. dissertation, series 18.2002, Copenhagen Business School, Denmark.

McConnell J.J., Servaes H. (1990), Additional evidence on equity ownership and corporate value. Journal of Financial Economics 27 (2), pp 595 -612.

Medina, J., y R. Valdés (1998), Flujo de Caja y decisiones de inversión en Chile: Evidencia de sociedades anónimas abiertas. Cuadernos de Economía 35(106): 301-323.

Megginson (1990), "Restricted Voting Stock, Acquisition Premiums, and the Market Value of Corporate Control", The Financial Review, 25, para Inglaterra.

Morck R., Shleifer A., Vishny R. (1989), Management ownership and market valuation: an empirical analysis. Journal of Financial Economics 20 (1), pp 293 -315.

Morck, R., A. Shleifer y R. Vishny (1988), "Management Ownership and Market Valuation, Journal of Financial Economics, enero.

Nickel S., Nicolitsas D., Dryden N. (1997), What makes firm perform well? European Economic Review 41 (3), pp $783-796$.

Paredes, R. y J. M. Sánchez (1994), “Grupos Económicos y Desarrollo: El Caso de Chile”. Ilades- Georgetown. Mimeo. 
Parisi, F., R. Godoy y A. Parisi (2000), Gobierno Corporativo en Chile: Evidencia. Facultad de Ciencias Económicas y Administrativas Universidad de Chile. Santiago, Marzo.

Rajan, R. y L. Zingales (1998), Financial dependence and growth, American Economic Review 88, 559-586.

Shleifer A., Vishny R. (1986), A Survey of Corporate Governance. Journal of Finance 52, pp 737-783.

Short H. (1994), Ownership, control, financial structure and the performance of firms. Journal of Economic Surveys 8 (3), pp 203-249.

Starks L. (2000), Corporate Governance and Institutional Investors: Implications for Latin America. Revista ABANTE, Vol 2, № 2, octubre.

Stulz R. (1988), Managerial control of voting rights: Financing policies and the market for corporate control, Journal of Financial Economics 20, pp 25-54.

Tobin J. (1969), “A General Equilibrium Approach to Monetary Theory," Journal of Money, Credit and Banking, 1, 15-29.

Thomsen S., Pedersen T. (2000), Ownership structure and Economic Performance in the largest European Companies. Strategic Management Journal, 21, pp 689-705.

Williamson, O. E. (1985), “The Economic Institutions of Capitalism,” New York: The Free Press.

Wolfenzon, D. (1999), A theory of pyramidal structures. Unpublished working paper. Harvard University: Cambridge, MA.

Zingales (1994), "The Value of the Voting Right: A Study of the Milan Stock Exchange Experience", Review of Financial Studies, 7.

Zingales (1995), "What determines the Value of Corporate Votes?, Quarterly Journal of Economics, 110.

Zingales, L. (1997), “Corporate Governance”, NBER WP 6309. 